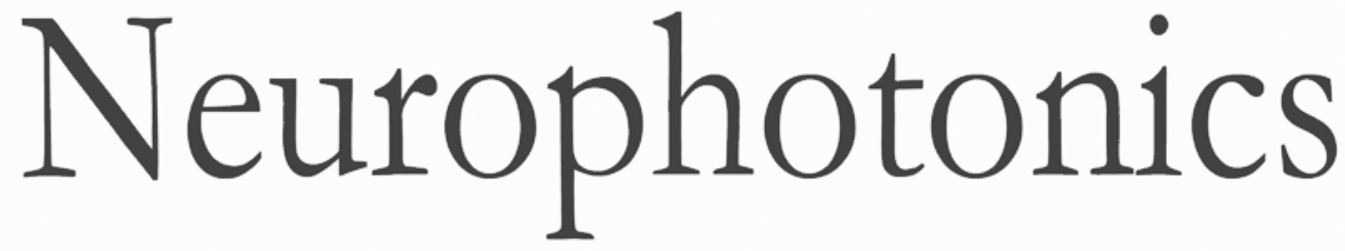

\title{
Detecting and measuring areas of choriocapillaris low perfusion in intermediate, non-neovascular age- related macular degeneration
}

\author{
Acner Camino \\ Yukun Guo \\ Qisheng You \\ Jie Wang \\ David Huang \\ Steven T. Bailey \\ Yali Jia
}




\title{
Detecting and measuring areas of choriocapillaris low perfusion in intermediate, non-neovascular age-related macular degeneration
}

\author{
Acner Camino, Yukun Guo, Qisheng You, Jie Wang, David Huang, Steven T. Bailey, and Yali Jia* \\ Oregon Health and Science University, Casey Eye Institute, Portland, Oregon, United States
}

\begin{abstract}
Age-related macular degeneration (AMD) is a vision-threatening disease that affects the outer retina and choroid of elderly adults. Because photoreceptors are found in the outer retina and rely primarily on the trophic support of the underlying choriocapillaris, imaging of flow or lack thereof in choriocapillaris by optical coherence tomography angiography (OCTA) has great clinical potential in AMD assessment. We introduce a metric using OCTA, named "focal perfusion loss" (FPL) to describe the effects of age and non-neovascular AMD on choriocapillaris flow. Because OCTA imaging of choriocapillaris is vulnerable to artifacts-namely motion, projections, segmentation errors, and shadows-they are removed by postprocessing software. The shadow detection software is a machine learning algorithm recently developed for the evaluation of the retinal circulation and here adapted for choriocapillaris analysis. It aims to exclude areas with unreliable flow signal due to blocking of the OCT beam by objects anterior to the choriocapillaris (e.g., drusen, retinal vessels, vitreous floaters, and iris). We found that both the FPL and the capillary density were able to detect changes in the choriocapillaris of AMD and healthy age-matched subjects with respect to young controls. The dominant cause of shadowing in AMD is drusen, and the shadow exclusion algorithm helps determine which areas under drusen retain sufficient signal for perfusion evaluation and which areas must be excluded. Such analysis allowed us to determine unambiguously that choriocapillaris density under drusen is indeed reduced. $\odot$ The Authors. Published by SPIE under a Creative Commons Attribution 4.0 Unported License. Distribution or reproduction of this work in whole or in part requires full attribution of the original publication, including its DOI. [DOI: 10.1117/1.NPh.6.4.041108]
\end{abstract}

Keywords: optical coherence tomography angiography; choriocapillaris; age-related macular degeneration; drusen.

Paper 19058SSRR received Jun. 8, 2019; accepted for publication Aug. 16, 2019; published online Sep. 12, 2019.

\section{Introduction}

Optical coherence tomography angiography (OCTA $)^{1,2}$ is an imaging modality that allows depth-resolved and noninvasive imaging of the retinal and choroidal flow. ${ }^{3-7}$ OCTA algorithms use the variation of optical coherence tomography (OCT) signal over time to detect blood flow against avascular tissue. Although significantly attenuated by the highly scattering retinal pigment epithelium (RPE), the OCT signal incoming from the choriocapillaris layer is generally enough to retrieve the blood flow information in both spectral domain and swept source OCT. Although the resolution of current OCTA devices is not sufficient to resolve either the true caliber of choroidal capillaries or the minuscule intravascular spaces, ${ }^{8}$ signal defects such as flow voids are large enough to be detected and measured. ${ }^{5,7}$

To date, numerous studies have been dedicated to quantifying reduced choriocapillaris flow automatically., $3,9,10$ Despite the potential clinical applications, quantification of flow impairments is challenged by the prevalence of different types of artifacts: projections, segmentation errors, motion, and shadows. Reluctance or inability to remove the projections of overlying retinal flow conduces to significant inaccuracies in interpretation and quantification of in situ flow. Erroneous segmentation of the very thin choriocapillaris layer can also produce incorrect visualization, the reason for which some previous studies have preferred to project a slab that is anatomically inaccurate but robust to segmentation errors. ${ }^{11,12}$ Motion artifacts due to

*Address all correspondence to Yali Jia, E-mail: jiaya @ohsu.edu saccades, ocular tremors or drift add a noise component that is difficult to subtract. Additionally, the choriocapillaris layer is vulnerable to signal loss due to severe shadowing by retinal vessels, large drusen, pupil vignetting, or vitreous floaters. Some of the previous works concerning choriocapillaris imaging with OCTA have excluded the entire area underneath drusen or large retinal vessels for quantitative analysis. Because commercially available fields of view are already limited, it is desirable to maximize the analytic area by accurately segmenting the extent of the shadowed pixels, retrieving signal that would have been ignored otherwise.

In this paper, we use a comprehensive artifact removal software to visualize the choriocapillaris in OCTA, including projection-resolved OCTA (PR-OCTA), segmentation of the anatomically accurate choriocapillaris slab by bidirectional graph search, and iterative, regression-based bulk motion subtraction. ${ }^{13}$ As a new addition to the advances made previously by our group, we adapt herein a shadow exclusion algorithm, previously developed for shadows originating in vitreous and anterior segment, ${ }^{14}$ which is trained here to add detection of shadows produced in the retina itself by large vessels and drusen. After artifact removal, we introduced a parameter to evaluate the choriocapillaris in low-perfusion areas (LPA), named focal perfusion loss (FPL). The LPA were the regions of earliest choriocapillaris perfusion impairment, detected by their reduced local capillary density with respect to baseline. With FPL, we account for reduced flow signal values over the LPA, which might be a signature of anomalous choriocapillaris function. 


\section{Method}

\subsection{Study Population}

This retrospective study included three groups recruited at the Casey Eye Institute of Oregon Health and Science University (OHSU). The first group consisted of subjects diagnosed with dry age-related macular degeneration (AMD) and intermediatesized drusen, excluding subjects with geographic atrophy (GA) or neovascular AMD. The second consisted of age-matched healthy subjects, and the third group was of young, healthy volunteers. Subjects in the AMD group were diagnosed by color fundus photography and OCT showing evidence of drusen and no evidence of choroidal neovascularization (CNV). Scans with signal strength index lower than 55 were excluded from analysis. The Institutional Review Board/Ethics Committee of OHSU approved the protocol and the research adhered to the tenets of the Declaration of Helsinki.

\subsection{Data Acquisition}

One eye of each participant was imaged with the AngioVue (RTVue-XR, Optovue, Inc. Fremont, California) spectral-domain OCT/OCTA system. Scans covered an area of $3 \times 3 \mathrm{~mm}^{2}$ centered at the fovea. Each scan consisted of two orthogonal acquisitions in horizontal and vertical priority directions registered into a single-volumetric data cube by Optovue's proprietary software. ${ }^{15}$ The isotropic sampling density was $\sim 10 \mu \mathrm{m} /$ pixel. The real-time eye tracking system incorporated in the AngioVue machine ${ }^{16}$ was used to minimize the prevalence of microsaccadic artifact residuals contaminating the flow signal. Two repeated B-scans were acquired at each location. Structural OCT images were generated by averaging B-scans at the same position. Blood flow images were generated using the commercial version of split-spectrum amplitude decorrelation angiography algorithm, which computes the decorrelation between two consecutive structural B-scans. ${ }^{17}$

\subsection{Data Preprocessing}

Four retinal layer boundaries (i.e., the vitreous/inner limiting membrane (ILM), outer plexiform layer (OPL)/outer nuclear layer (ONL), RPE and Bruch's membrane/choriocapillaris) were segmented from structural OCT B-scans by a validated directional graph search method. ${ }^{18}$ An iterative regression-based bulk motion subtraction (irb-BMS) algorithm ${ }^{19}$ was used to remove the confounding contribution of ocular motion. By application of irb-BMS, nonvascular voxels in the retina and choriocapillaris could be identified and removed in a thresholding scheme that sets a reflectance-adjusted threshold based on the regression analysis of background OCTA signal versus local reflectance, retrieving flow signal in pixels in areas of partial attenuation. Despite this feature, when the OCT signal is largely attenuated, OCTA flow signal is filtered out and cannot be retrieved by irb-BMS. This is the case of shadows cast by opacities anterior to the tissue of interest, for which we have previously trained a separate segmentation software using retinal images and we adapt here with choriocapillaris images. Projection

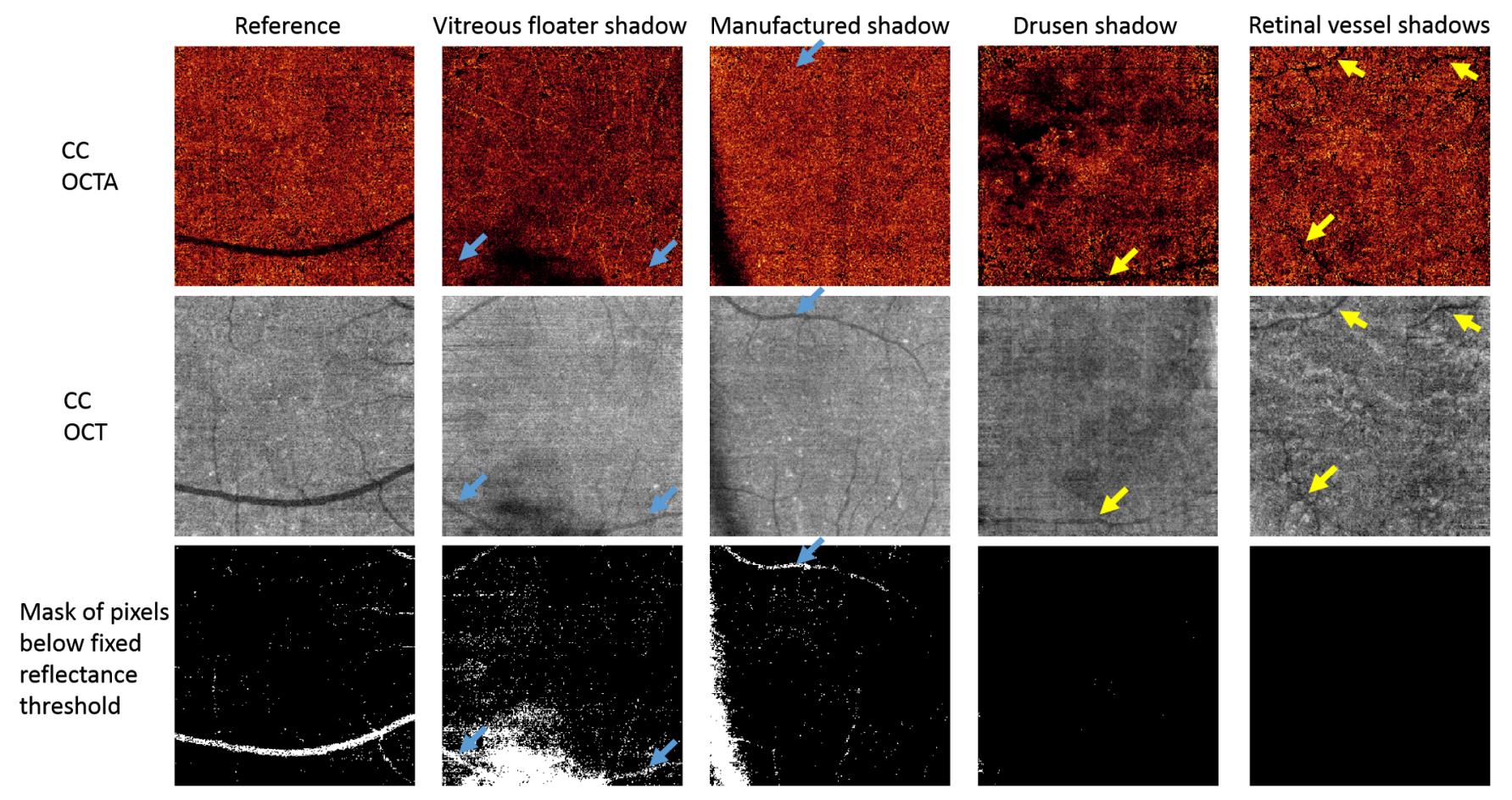

Fig. 1 Inability to successfully identify shadowed pixels by simply using a fixed reflectance threshold. Five cases are shown: a reference image from a young, healthy subject and four other images representing different sources of shadows (vitreous floaters, a manufactured shadow by a polylactic filament partially blocking the scanning beam, drusen, and retinal vessels). A fixed reflectance threshold was applied to the en face OCT of the choriocapillaris slab to detect shadow areas and the results shown in the bottom row. The fixed threshold generally identifies shadow areas with artefactual reduction in choriocapillaris density, but it sometimes identifies areas with normal choriocapillaris density that does not need to be excluded (blue arrows) and sometimes fails to detect shadow areas with artefactually reduced choriocapillaris density (yellow arrows). 
artifacts cast by inner retinal flow onto the outer retina and choriocapillaris were removed by a reflectance-based PR-OCTA algorithm. ${ }^{20}$ Then in situ flow at different retinal depths could be recognized. En face angiograms of the inner retinal blood flow were generated by maximum projection of the flow signal between vitreous/ILM and OPL/ONL interfaces. En face angiograms of the choriocapillaris were generated by maximum projection of a slab located between 10 and $18 \mu \mathrm{m}$ below the Bruch's membrane, considering the RPE-to-choriocapillaris distance reported recently for the normal population. ${ }^{8}$

\subsection{Automatic Detection of Shadows on Choriocapillaris Flow}

Primarily, shadows appear in OCTA because the speckle variance signal in very dark pixels is filtered out by the OCTA algorithm. But contrary to one's expectation, the reflectance of voxels is not enough to successfully identify shadowed areas. In our previous work where we segmented shadows in the inner retina, we showed that many times dark pixels are caused by reduced tissue reflectivity related to the pathology and not by anterior opacities (e.g., vitreous floaters). Thus simply analyzing the reflectance of the choriocapillaris slab is not an accurate way to identify areas with shadow artifacts (Fig. 1). An approach that analyzes both reflectance and flow information is needed. We chose to do so with a machine learning algorithm that could account for the complex interaction between reflectance and flow signals.
A supervised machine learning algorithm similar to the reported previously for shadows on retina ${ }^{14}$ was trained to detect the shadows on choriocapillaris. Briefly, it consisted of an ensemble learning method (RUS-Boost trees) that used features of both reflectance and flow information, which were normalized to the average of the normal population. Four features were used: the mean local reflectance, mean local flow index (averaged OCTA flow signal), and standard deviations (SD) of reflectance and flow signal in the A-line direction for a 10-pixel thickness choroidal slab below the Bruch's membrane (Fig. 2). The SD feature was included, because it could help distinguish reduced tissue reflectivity from shadow. Reduced tissue reflectivity generally reduces the mean but not the SD of reflectance signal. In contrast, shadows reduce the mean and SD of both reflectance and flow signals.

In order to generate reliable labels for the shadowed areas, we created manufactured shadows on scans of 10 young, healthy subjects (age $31 \pm 4$ years old) by partially blocking the scanning beam (Fig. 2) with a filament of polylactic acid placed between the cornea and the OCTA instrument and by reducing the diameter of an external iris that creates vignetting in the corners of images. A total of 924,160 points and their labels (shadowed versus nonshadowed) composed the training dataset. Labeling of the manufactured shadow positions was done by thresholding the position-dependent change in the local vessel density [Fig. 2(b)] with respect the variation of the same variable in two reference scans of the same subject acquired under optimal imaging conditions. ${ }^{14}$ This objective labeling mechanism is
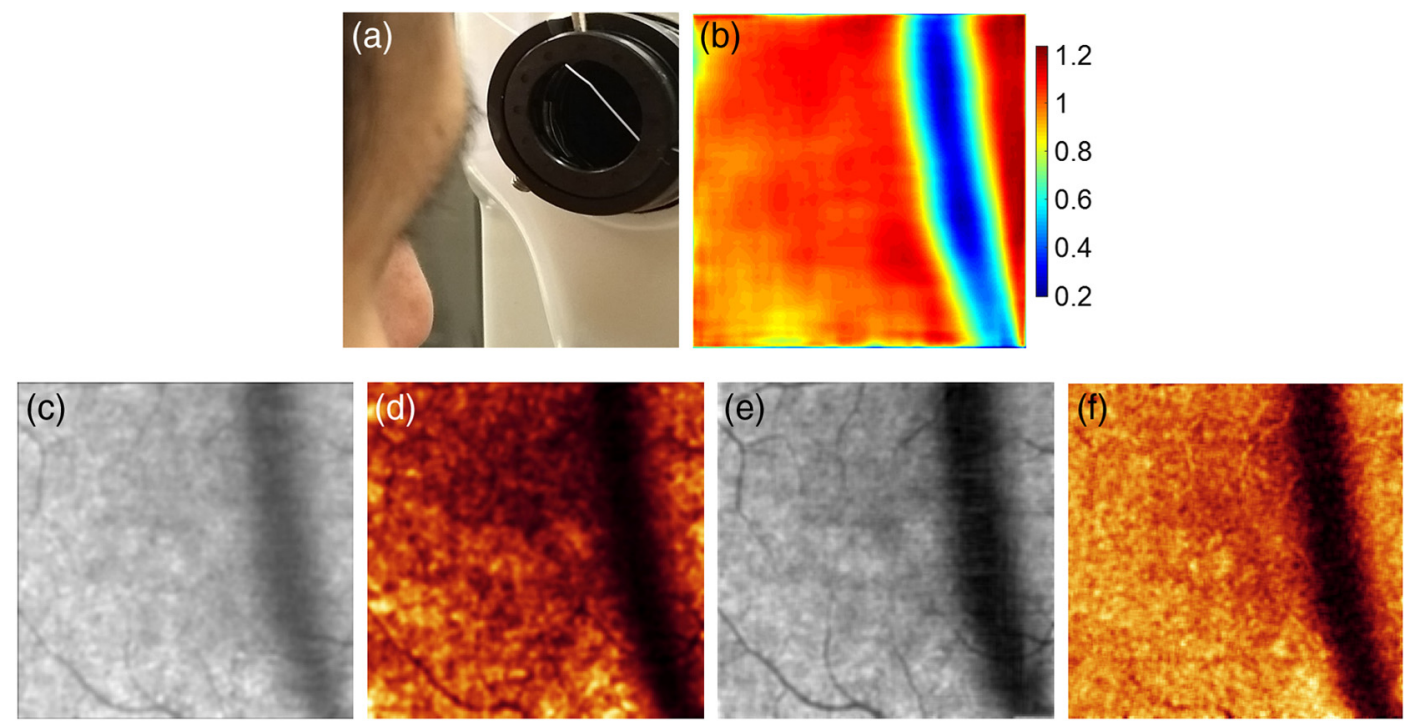

Fig. 2 Features of the shadow detection algorithm used to detect areas of choriocapillaris with unreliable flow signal. A supervised machine learning algorithm (a forest of decision trees) was trained on images from manufactured shadows on healthy subjects (a) using four features from both OCT and OCTA volumetric data. A RUS-boost modality (a hybrid of random undersampling of the majority class and boosting) was used to alleviate the class imbalance. The labels were assigned based on the variation of the local vessel density compared to the variation in two scans acquired from the same healthy subject under optimal imaging conditions. ${ }^{14}$ The position-wise ratio of registered local vessel densities between a shadowed and a reference scan of the same eye is represented in (b). The features used to train the forest were average reflectance (c) and average flow signal (d) in the vicinity of each pixel, as well as SD of the reflectance (e) and the flow signal (f) along the A-line direction from the Bruch's membrane in a 20-pixel thickness slab, exploiting the fact that shadows are dark structures that propagate forward in the axial direction. More details of the algorithm in its retinal implementation and the validation on healthy subjects can be found in our previous publication. ${ }^{14}$ 

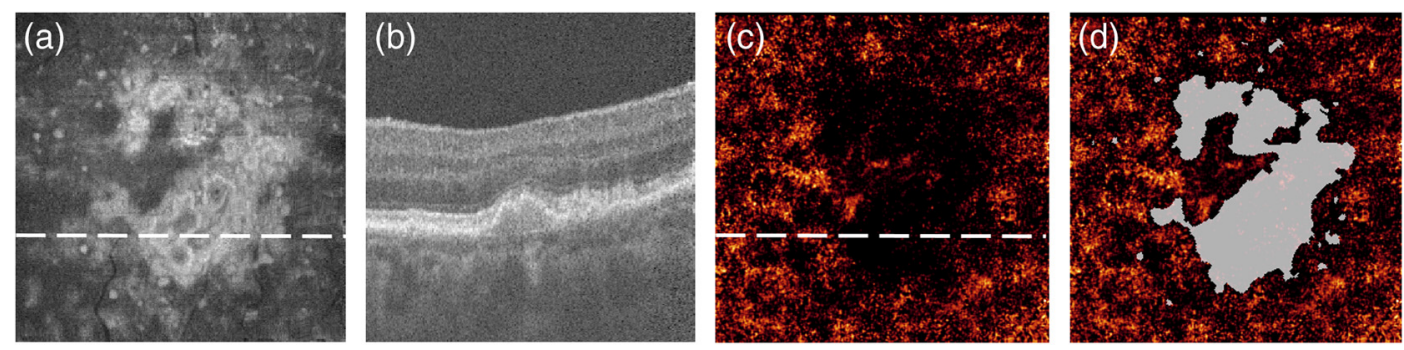

Fig. 3 Automatic detection of drusen in eyes with dry age-related macular degeneration: (a) en face projection of the OCT reflectance within a slab where drusen is found; (b) representative B-scan containing drusen, indicated by the position marked with white dashed lines; (c) maximum projection of the flow within the choriocapillaris flow; and (d) mask of drusen area overlaid on the choriocapillaris angiogram.

very reliable as these healthy subjects are known to have uniform normal choriocapillaris density.

\subsection{Drusen Detection}

Drusen is an important cause of shadows on OCTA of the choriocapillaris in subjects with AMD. A previous drusen detection method was applied to AMD scans (Fig. 3). ${ }^{21}$ This method used slabs carefully selected at certain distances from the Bruch's membrane in order to delineate the boundaries of pathological areas with minimum layer segmentation requirements. After finding the drusen area, the RPE segmentation was further used to estimate height at every drusen position. The software can detect soft drusen, cuticular drusen, and subretinal drusenoid deposits (also called pseudodrusen in bibliography) but cannot differentiate between the three categories. The drusen and shadow detection algorithms were entirely independent of each other.

\subsection{Quantitative Evaluation of Choriocapillaris Perfusion}

After irb-BMS, en face angiograms of the in situ flow showed zero value at nonvascular positions. The capillary density was defined as the percentage of vascular pixels through the whole en face angiograms excluding shadowed areas.

Although capillary density can be used to assess overall tissue perfusion, we propose here the FPL as an additional metric to describe OCTA signal in areas of focally reduced perfusion. For this purpose, vascular binary maps of the choriocapillaris were first generated from scans of 40 young healthy eyes. Then local density maps $D_{\text {map }}$ were generated by computing the capillary density within a surrounding $11 \times 11$-pixel area for each lateral position (Fig. 4). An expert grader manually selected the center of the foveal avascular zone (FAZ), and all local density maps were registered by rigid translations to make their FAZ-center positions overlap. At every position of the

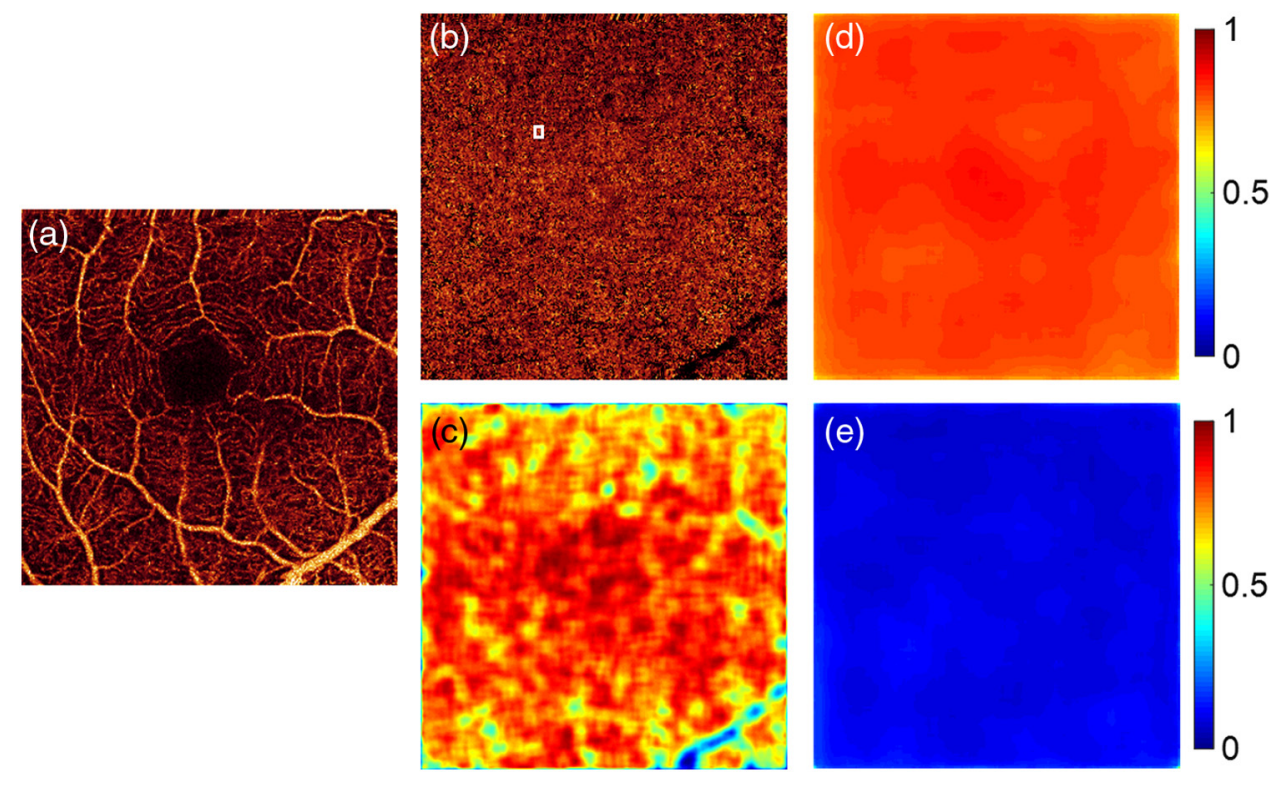

Fig. 4 Generation of choriocapillaris perfusion density maps from healthy subjects. (a) En face projection of the superficial vascular complex. (b) Choriocapillaris layer with projection and motion artifacts removed. The white square represents the kernel size used to generate density maps. (c) Density maps were generated by assigning to each pixel the local capillary density within the area enclosed by a moving window of size $11 \times 11$ pixels [white box in (b)]. (d) Average of density maps from 40 scans on young, healthy eyes after registration to overlap their FAZ centers. Choriocapillaris vessel density was relatively homogeneous on this macular window. (e) SD of 40 density maps from young, healthy eyes after registration to overlap their FAZ centers. 

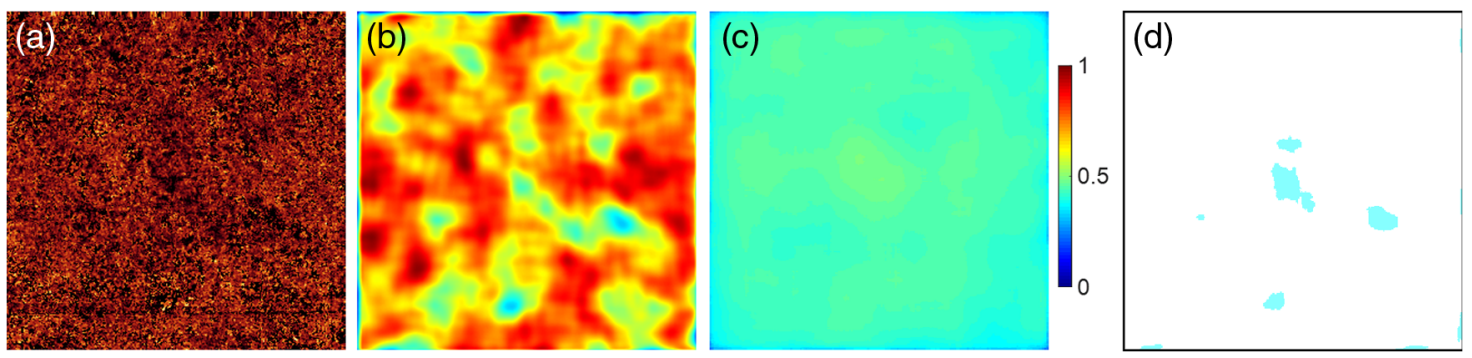

Fig. 5 Choriocapillaris LPA from a subject in the age-matched control group: (a) en face projection of choriocapillaris flow; (b) perfusion density map; (c) reference threshold map generated from young, healthy controls by $\mathrm{CC}_{\text {ref }}(x, y)-3.1 \times \mathrm{CC}_{\text {std }}(x, y)$ [Figs. 4(d) and 4(e)]; and (d) LPA map generated by recognizing the positions of local capillary density values in (b) below threshold in (c). The areas of apparent perfusion loss in (d) can be owing to either choriocapillaris dropout or flow signal loss in shadowed areas. Shadowed areas detected previously were excluded from LPA.

registered local density maps, the average and SD of the capillary density values were used to generate reference maps $\mathrm{CC}_{f f}$ and $\mathrm{CC}_{\text {std }}$, respectively, describing the normal population [Figs. 4(c) and 4(d)]. LPA were defined by superpixel capillary density below the 0.1 percentile threshold in the normal reference eyes, i.e., threshold $(x, y)=\mathrm{CC}_{\text {ref }}(x, y)-3.1 \mathrm{CC}_{\text {std }}(x, y)$ (Fig. 5). The distribution of contiguous LPA in young controls was examined and its 99 percentile cutoff point -15 grouped pixels $\left(0.001 \mathrm{~mm}^{2}\right)$ - was used as the contiguity threshold. The LPA in each eye should meet both low perfusion and contiguity requirements.

Once the LPA were found, the FPL was defined as the choriocapillaris density loss (relative to the normal reference) integrated over the LPA and then expressed as the percentage of the
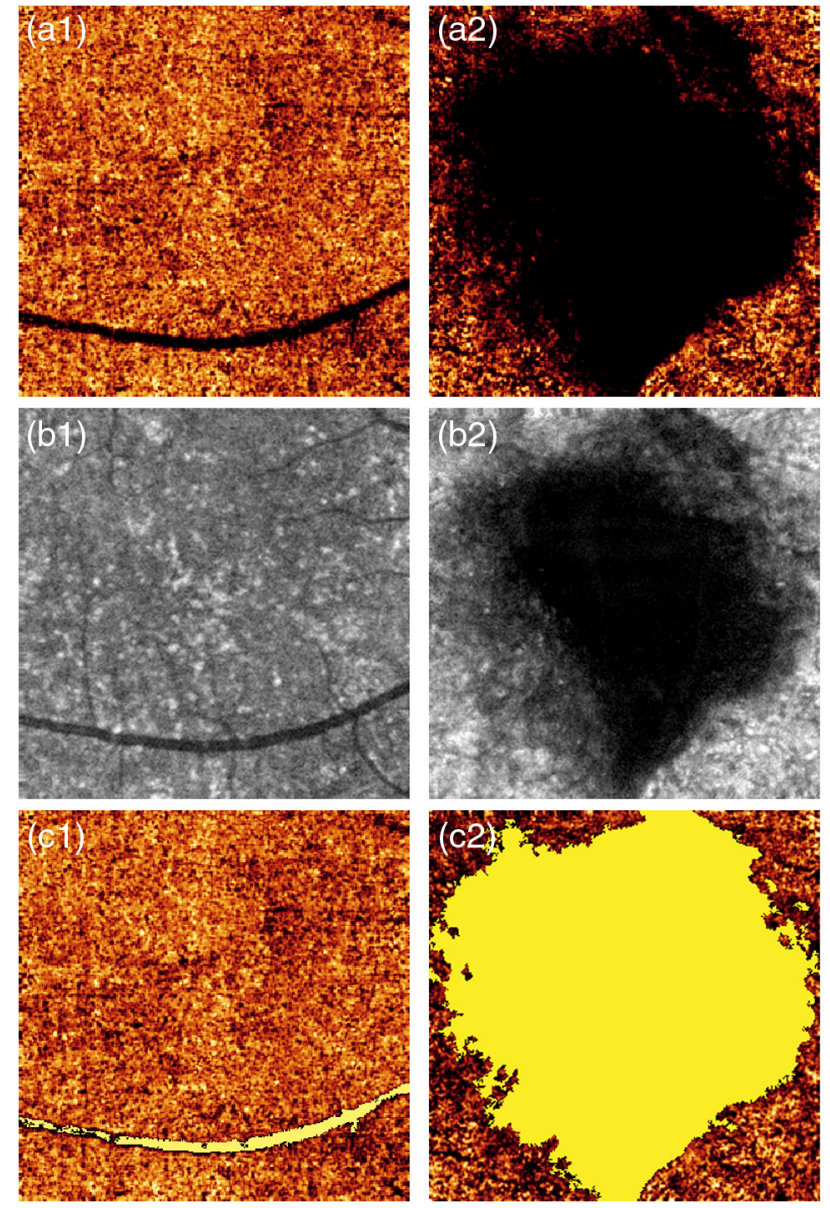
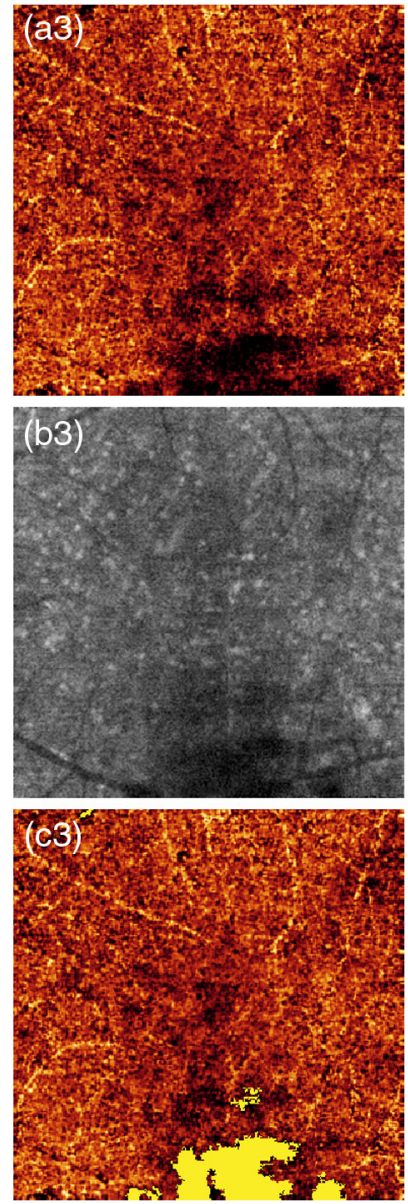

Fig. 6 Three representative examples of shadow detection performance: (1) young healthy subject with a large retinal vessel casting a shadow on choriocapillaris, (2) age-related macular degeneration subject with a large drusen shadow, and (3) subject without AMD but with a large vitreous floater casting shadows on retina and choriocapillaris. (a) En face projections of the flow signal in the choriocapillaris slab showing significant absorption from anterior opacities, (b) corresponding mean reflectance projections of the choriocapillaris slab, and (c) results of the shadow detection overlaid on choriocapillaris angiogram. 
choriocapillaris density integral of the normal reference map with shadows excluded:

$\mathrm{FPL}=\left|\frac{\sum \sum_{\mathrm{LPA}}\left[\mathrm{CC}(x, y)-\mathrm{CC}_{\mathrm{ref}}(x, y)\right]}{\sum \sum_{\mathrm{AA}} \mathrm{CC}_{\mathrm{ref}}(x, y)}\right|$,

where $\mathrm{CC}$ is the density map of the AMD subject under analysis and AA is the analytic area.

The relationships of drusen area with either capillary density or FPL within the effective analytic area were investigated for AMD subjects.

A Wilcoxon rank sum test was used to evaluate the statistical significance between AMD, young, and age-matched healthy groups, determined by $p<0.05$.

\section{Results}

Choriocapillaris FPL and capillary density were calculated for forty young healthy eyes, 26 eyes with intermediate AMD, and 18 age-matched controls.

Shadows were detected for different absorptive elements superjacent to the choriocapillaris such as large retinal vessels [Figs. 6(a1)-6(c1)], drusen [Figs. 6(a2)-6(c2)], and vitreous floaters [Figs. 6(a3)-6(c3)]. In the AMD group, a large portion of the areas detected as shadows correlated spatially with higher drusen height (Fig. 7). Drusen area of AMD subjects was $15.0 \%$ of the total area but the shadows were only $8.4 \%$ (Table 1 ), allowing a large portion of the areas under drusen to be analyzed for perfusion loss. LPA were found both under and around drusen. After exclusion of shadows, cumulative LPA were found to be larger than drusen area $\left(2.7 \pm 1.7 \mathrm{~mm}^{2}\right.$ versus $1.4 \pm 0.7 \mathrm{~mm}^{2}$, $p<0.01)$. Shadow area in the young and age-matched groups only represented $0.1 \%$ and $0.04 \%$ of the total scanning area.

There was FPL in all scans of the AMD and age-matched groups and in $77.5 \%$ of the young healthy group. FPL was larger in the AMD group compared to the age-matched and young control groups (Table 1, Fig. 8). The larger FPL found in old controls compared to young controls is in agreement with a recent report showing increased choriocapillaris flow deficits with age, measured with swept-source OCTA. ${ }^{22}$ In terms of capillary density, the AMD group showed a significantly smaller value than the age-matched $(p<0.01)$ and the young $(p<0.01)$ healthy control groups (Table 1). Capillary density of the young control group was also larger than the age-matched control group $(p<0.01)$. For both healthy reference groups, the capillary density was independent from the OCT signal strength $(R=$ $-0.13, p=0.63$, age-matched and $R=0.20, p=0.16$, young). Capillary density was lower under drusen than in areas with
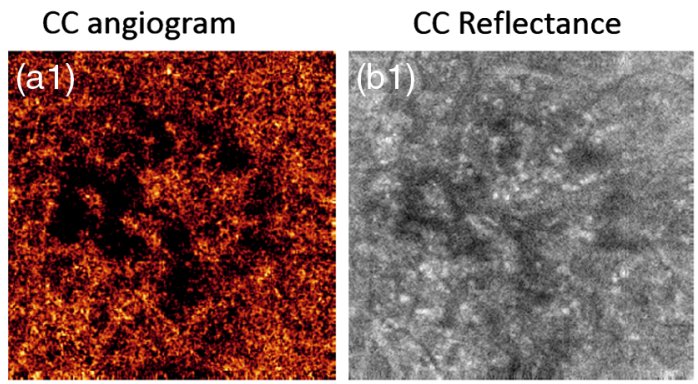
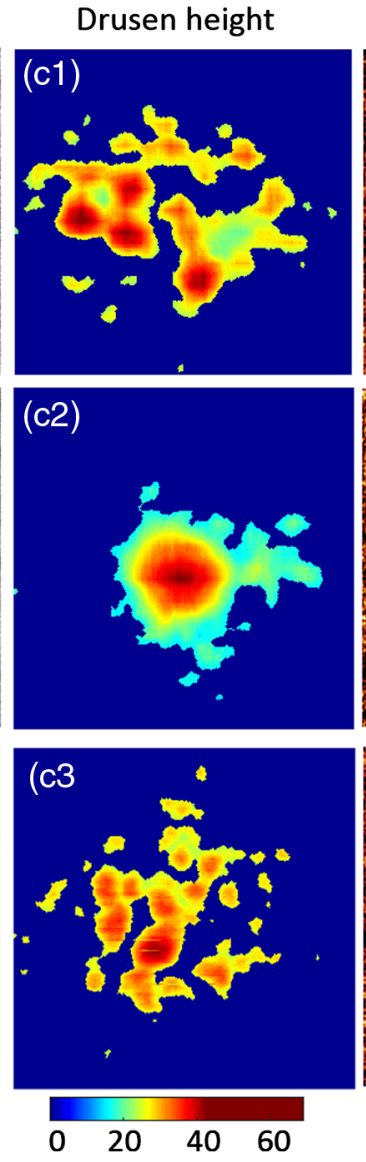

\section{Shadow overlay}
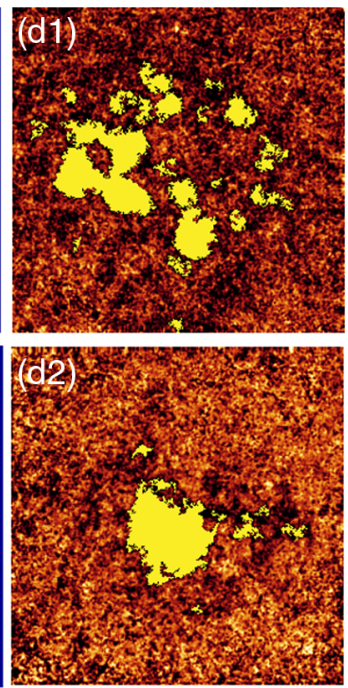

LPA \& shadow overlay
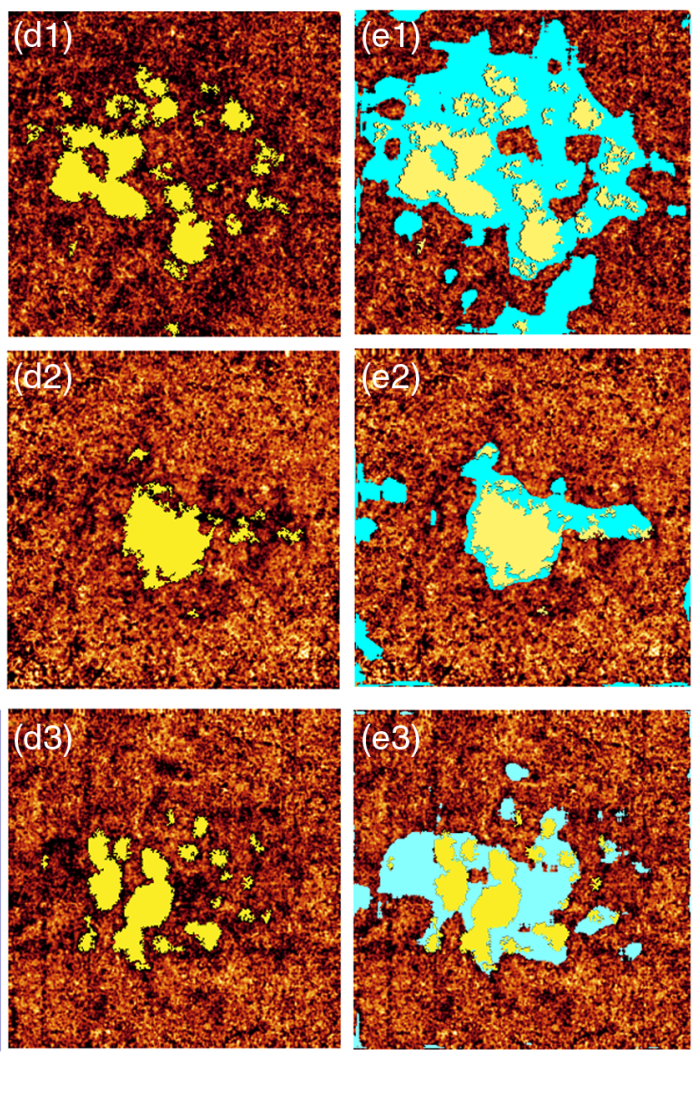

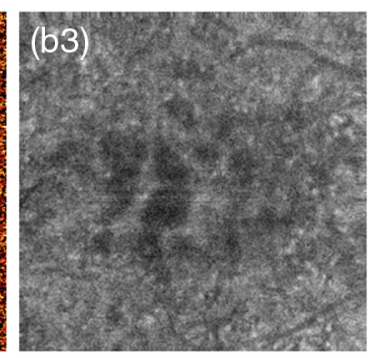

Fig. 7 Comparison of the location of drusen areas versus shadow areas in three AMD subjects. (a1)-(a3) The en face OCTA image of the choriocapillaris layer; (b1)-(b3) the corresponding reflectance image. (c1)-(c3) Both perfusion loss and preserved perfusion areas are apparent under drusen. (d1)-(d3) The positions detected as unreliable, shadowed OCTA signal in yellow, overlaid on the en face angiograms. These shadows were typically located at the positions of large drusen height. (e1)-(e3) The LPA detected in blue. 
Table 1 Differences between the three groups recruited in this study (mean $\pm \mathrm{SD}$ ). Capillary density of young controls was larger $(p<0.01)$ and FPL was lower $(p<0.01)$ than age-matched ones. Capillary density of AMD was lower $(p<0.01)$ and FPL was larger $(p<0.01)$ than age-matched controls.

\begin{tabular}{|c|c|c|c|c|}
\hline Group & $\begin{array}{c}\text { Age } \\
\text { (years) }\end{array}$ & $\begin{array}{l}\text { Shadow } \\
\text { exclusion } \\
\text { area } \\
\text { (\% area) }\end{array}$ & $\begin{array}{c}\text { Capillary } \\
\text { density } \\
(\% \text { area })^{*}\end{array}$ & FPL (a.u.) ${ }^{*}$ \\
\hline Young controls & $31 \pm 5$ & $0.1 \pm 0.4$ & $80.5 \pm 7.3$ & $0.01 \pm 0.04$ \\
\hline $\begin{array}{l}\text { Age-matched } \\
\text { controls }\end{array}$ & $75 \pm 5$ & $0.04 \pm 0.1$ & $68.1 \pm 6.3$ & $0.04 \pm 0.04$ \\
\hline AMD & $81 \pm 2$ & $8.4 \pm 7.8$ & $54.5 \pm 8.6$ & $0.29 \pm 0.19$ \\
\hline
\end{tabular}

"Statistical significances of the differences between groups were evaluated by a Wilcoxon rank-sum test.

healthy RPE ( $p<0.05)$, suggesting that although LPA typically surround the drusen, FPL is more severe under drusen than outside of it.

\section{Discussion}

In this study, we have investigated perfusion defects of the choriocapillaris in elderly and AMD subjects using OCTA. Instead of finding choriocapillaris flow voids by applying various thresholding mechanisms such as Al-Sheikh et al., ${ }^{23}$ Borrelli et al., ${ }^{24}$ and Spaide, ${ }^{10}$ we proposed a new metric called FPL. This quantity accounts for low-focal perfusion density surrounding choriocapillaris pixels as well as the OCTA flow signal of pixels, whereas the capillary density accounts for the percentage of vascular pixels in the whole image, FPL is a ratio of the flow signal value in LPA to the total flow signal. It allows one to differentiate mild homogeneous capillary dropout over the entire field of view with no LPA — and thus zero FPL—from small but severe "islands" of focal capillary dropout.

Evidence of drusenoid deposits has been, for a long time, the earliest clinical sign of AMD due to their easy detectability in a fundus exam. Drusen obstructs the metabolic exchange between choriocapillaris and the outer retina, a process than can advance into vision-threatening stages of AMD known as $\mathrm{GA}^{25}$ and $\mathrm{CNV}^{26}$ With the advent of OCTA, imaging of the choroidal flow in a noninvasive way was possible, giving access to study early vascular effects of the disease and their possible relationship with drusen development. Appropriate interpretation of the choriocapillaris angiogram will help answer the question of whether there is a causal link between drusen deposits and choriocapillaris ischemia. Therefore, accurate exclusion of the areas with unreliable OCTA signal on choriocapillaris angiograms is crucial. This problem has challenged all previous studies attempting quantification of choroidal flow anomalies in AMD. Recently, Zhang et al. ${ }^{9}$ and Nassisi et al. ${ }^{27,28}$ used methods that compensated the partial attenuation of choriocapillaris OCTA signal by drusen absorption, using the in situ OCT reflectance.
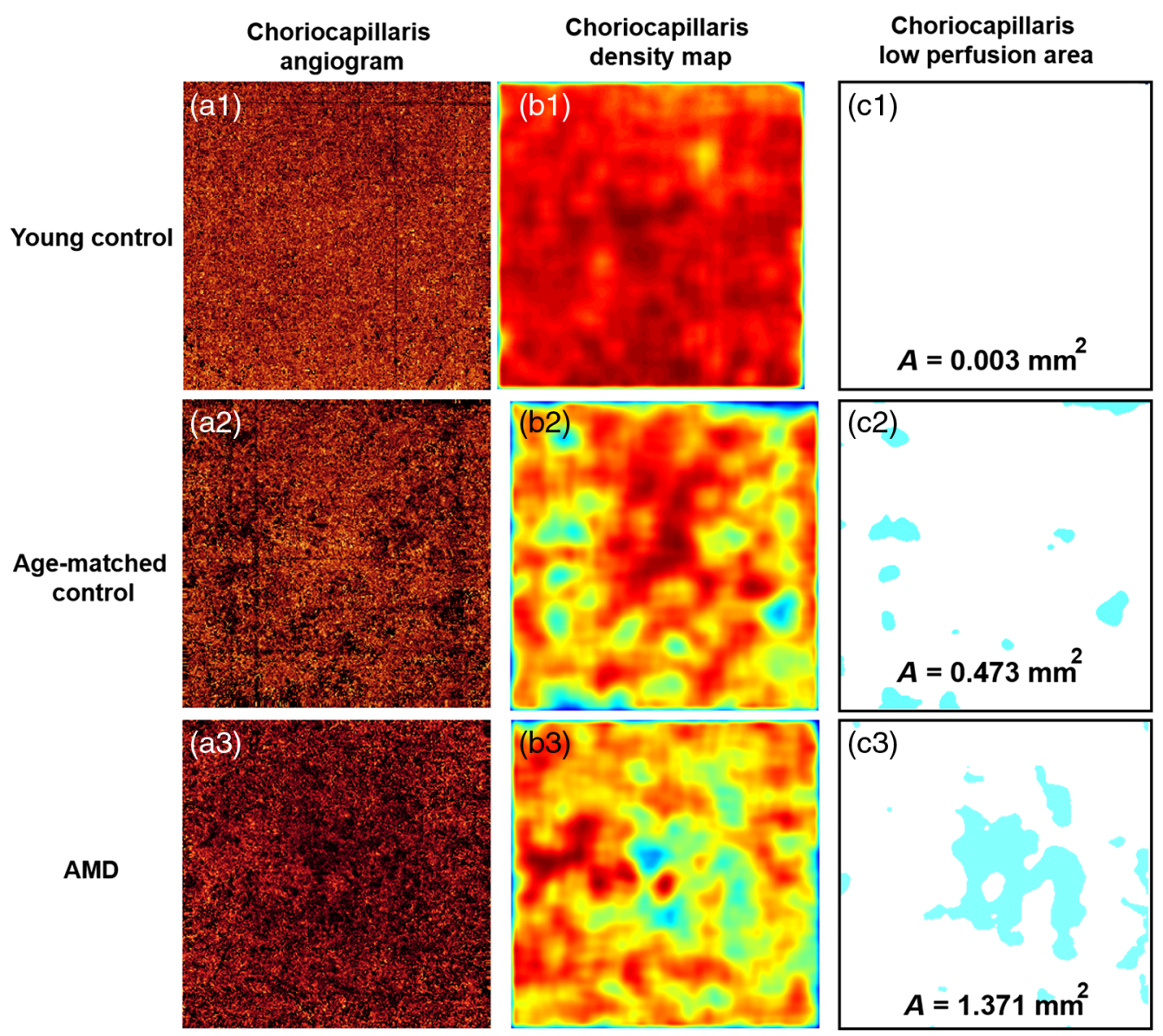

Fig. 8 Choriocapillaris en face angiograms, density maps, and LPA of (a1)-(c1) a young, healthy subject; (a2)-(c2) a control subject from the age-matched group; and (a3)-(c3) a dry AMD subject. 
Although choriocapillaris signal under drusen was amplified, large drusen would still block the backscattered light to such extent that exceeds the range of retrievable OCTA signal. Zhang et al. used an empirical threshold value at less than two times the reflectance value in the FAZ region to detect these areas. Conversely, we proposed here a machine learning algorithm that does not rely on reflectance only to detect the severe shadowing. Our experiment on manufactured shadowing helped us generate labels devoid of human subjectivity prior to training and to estimate the performance of the algorithm, which is otherwise impossible to evaluate using clinical shadows.

Although the three-dimensionality of OCTA offers great potential, artifacts are abundant. In our study, we have removed the contribution of projection and bulk motion artifacts from the choriocapillaris slab and excluded shadowed areas. Taking measures to reduce these artifacts by image processing should be a critical step in any study attempting to interpret the OCTA signal in the choriocapillaris layer. Previously, choriocapillaris capillary densities of normal population have been reported in the range of $90 \%$ to $99 \%$ in OCTA studies that did not make efforts to remove projection or bulk motion artifacts, ${ }^{29-31}$ overestimating the real choriocapillaris capillary density. The macular choriocapillaris density of $\sim 80 \%$ found in our work is a closer estimation to the value reported by histology. ${ }^{32}$

The choriocapillaris consists of a very thin network of capillaries located very close to the Bruch's membrane. However, it has become a common practice to project the choriocapillaris slab slightly below the anatomically correct slab, ${ }^{11,12}$ generating images formed by projected flow rather than in situ flow. This solution attempts to prevent the confounding effect of amplified retinal projections and motion artifacts that might appear in the event of layer segmentation errors. ${ }^{5,11}$ Using PR-OCTA algorithm, the irb-BMS algorithm, and the accurate layer segmentation of our graph-search method, ${ }^{18}$ we could visualize the choriocapillaris at its anatomically accurate slab depth.

There are a few limitations to this study. Because the shadow detection algorithm had to be trained with data from manufactured shadows on healthy subjects in order to produce reliable training labels, the size of the available dataset is small. Adding natural vitreous shadows from healthy subjects to the training dataset could potentially enhance the forest performance. Labeling of shadow boundaries could be possible if vitreous floaters spontaneously moved away from the field of view or if they were extracted (e.g., the case where shadows disappeared after a vitrectomy procedure in Ref. 14), but recruitment of these cases is very limited. There is an inherently large class imbalance in this method, but it was alleviated by the choice of a RUS-boost forest modality, where the majority class was randomly under-sampled during training. Finally, the small size of the groups recruited might limit the statistical significance of results.

\section{Conclusion}

In summary, we used OCTA to evaluate the choriocapillaris damage caused by the progression of age and AMD. Two metrics, capillary density and FPL, were investigated using in situ flow in dry AMD and age-matched subjects. Analysis was done after comprehensive artifact correction/exclusion that included projection, motion, and shadow artifacts. The FPL metric encompassed the extent of the damage as well as the degree of flow signal reduction and was useful to differentiate healthy from AMD subjects. We believe this metric has great potential to assess small changes in capillary perfusion in the early stages of AMD.

\section{Disclosures}

David Huang: Optovue Inc. (F, I, P, R). Yali Jia: Optovue Inc. (F, P). Acner Camino: Optovue Inc. (P). Oregon Health and Science University, Acner Camino, Yali Jia, and David Huang have financial interest in Optovue Inc., a company that may have a commercial interest in the results of this research and technology. These potential conflicts of interest have been reviewed and managed by OHSU. Other authors do not have financial interest in this article.

\section{Acknowledgments}

This work was supported by Grant Nos. R01 EY024544, R01EY027833, P30 EY010572, and T32 EY023211-05 from the U.S. National Institutes of Health (Bethesda, Maryland), an unrestricted departmental funding grant, the William and Mary Greve Special Scholar Award from Research to Prevent Blindness (New York), and the Antonio Champalimaud Vision Award.

\section{References}

1. Y. Jia et al., "Quantitative optical coherence tomography angiography of choroidal neovascularization in age-related macular degeneration," Ophthalmology 121(7), 1435-1444 (2014).

2. J. Ma et al., "Optical coherence tomographic angiography imaging in age-related macular degeneration," Ophthalmol. Eye Dis. 9, 117917211668607 (2017).

3. P. L. Nesper, B. T. Soetikno, and A. A. Fawzi, "Choriocapillaris nonperfusion is associated with poor visual acuity in eyes with reticular pseudodrusen," Am. J. Ophthalmol. 174, 42-55 (2017).

4. C. A. Moreira-Neto et al., "Choriocapillaris loss in advanced age-related macular degeneration,” J. Ophthalmol. 2018, 1-6 (2018).

5. J. L. Lauermann, N. Eter, and F. Alten, "Optical coherence tomography angiography offers new insights into choriocapillaris perfusion," Ophthalmologica 239(2-3), 74-84 (2018).

6. W. Choi et al., "Ultrahigh-speed, swept-source optical coherence tomography angiography in nonexudative age-related macular degeneration with geographic atrophy," Ophthalmology 122(12), 2532-2544 (2015).

7. R. F. Spaide, "Choriocapillaris flow features follow a power law distribution: implications for characterization and mechanisms of disease progression," Am. J. Ophthalmol. 170, 58-67 (2016).

8. K. Kurokawa, Z. Liu, and D. T. Miller, "Adaptive optics optical coherence tomography angiography for morphometric analysis of choriocapillaris [Invited]," Biomed. Opt. Express 8(3), 1803-1822 (2017).

9. Q. Zhang et al., "A novel strategy for quantifying choriocapillaris flow voids using swept-source OCT angiography," Invest. Ophthalmol. Visual Sci. 59(1), 203-211 (2018).

10. M. Nicolo et al., "Choroidal vascular flow area in central serous chorioretinopathy using swept-source optical coherence tomography angiography," Invest. Ophthalmol. Vis. Sci. 58(4), 2002-2010 (2017).

11. E. Moult et al., "Ultrahigh-speed swept-source OCT angiography in exudative AMD," Ophthalmic Surg. Lasers Imaging Retina 45(6), 496-505 (2014).

12. A. Matet et al., "Patterns of choriocapillaris flow signal voids in central serous chorioretinopathy: an optical coherence tomography angiography study," Retina Publish Ahead of Print (9000).

13. A. Camino et al., "Enhanced quantification of retinal perfusion by improved discrimination of blood flow from bulk motion signal in OCTA," Transl. Vision Sci. Technol. 7(6), 20 (2018).

14. A. Camino et al., "Automated detection of shadow artifacts in optical coherence tomography angiography," Biomed. Opt. Express 10(3), 1514-1531 (2019).

15. M. F. Kraus et al., "Motion correction in optical coherence tomography volumes on a per A-scan basis using orthogonal scan patterns," Biomed. Opt. Express 3(6), 1182-1199 (2012). 
16. A. Camino et al., "Evaluation of artifact reduction in optical coherence tomography angiography with real-time tracking and motion correction technology," Biomed. Opt. Express 7(10), 3905-3915 (2016).

17. Y. Jia et al., "Split-spectrum amplitude-decorrelation angiography with optical coherence tomography," Opt. Express 20(4), 4710-4725 (2012).

18. M. Zhang et al., "Advanced image processing for optical coherence tomographic angiography of macular diseases," Biomed. Opt. Express 6(12), 4661-4675 (2015).

19. A. Camino et al., "Regression-based algorithm for bulk motion subtraction in optical coherence tomography angiography," Biomed. Opt. Express 8(6), 3053-3066 (2017).

20. J. Wang et al., "Reflectance-based projection-resolved optical coherence tomography angiography [Invited]," Biomed. Opt. Express 8(3), 1536-1548 (2017).

21. R. Zhao et al., "Automated drusen detection in dry age-related macular degeneration by multiple-depth, en face optical coherence tomography," Biomed. Opt. Express 8(11), 5049-5064 (2017).

22. F. Zheng et al., "Age-dependent changes in the macular choriocapillaris of normal eyes imaged with swept-source optical coherence tomography angiography," Am. J. Ophthalmol. 200, 110-122 (2019).

23. M. Al-Sheikh et al., "Quantitative OCT angiography of the retinal microvasculature and the choriocapillaris in myopic eyes," Invest. Ophthalmol. Visual Sci. 58(4), 2063-2069 (2017).

24. E. Borrelli et al., "Alterations in the choriocapillaris in intermediate agerelated macular degeneration," Invest. Ophthalmol. Visual Sci. 58(11), 4792-4798 (2017).
25. F. G. Holz et al., "Geographic atrophy," Ophthalmology 121(5), 1079_ 1091 (2014).

26. W. R. Green and D. J. Wilson, "Choroidal neovascularization," Ophthalmology 93(9), 1169-1176 (1986).

27. M. Nassisi et al., "Choriocapillaris flow impairment surrounding geographic atrophy correlates with disease progression," PLoS One 14(2), e0212563 (2019).

28. M. Nassisi et al., "Choriocapillaris impairment around the atrophic lesions in patients with geographic atrophy: a swept-source optical coherence tomography angiography study," Br. J. Ophthalmol. 103, 911-917 (2019)

29. M. Nassisi et al., "Short-term choriocapillaris changes in patients with central serous chorioretinopathy after half-dose photodynamic therapy," Int. J. Mol. Sci. 18(11), 2468 (2017).

30. S. M. McClintic et al., "Quantitative optical coherence tomography angiography of the choriocapillaris in central serous chorioretinopathy," Invest. Ophthalmol. Visual Sci. 56(7), 3348-3348 (2015).

31. F. Alten et al., "Exploring choriocapillaris under reticular pseudodrusen using OCT-angiography," Graefes Arch. Clin. Exp. Ophthalmol. 254(11), 2165-2173 (2016).

32. R. S. Ramrattan et al., "Morphometric analysis of Bruch's membrane, the choriocapillaris, and the choroid in aging," Invest. Ophthalmol. Visual Sci. 35(6), 2857-2864 (1994).

Biographies of the authors are not available. 